
\title{
25 Research Suare \\ Yiqi Fumai Injection for patients with Septic Shock: study protocol for a randomized controlled trial 1
}

\section{jing zeng}

the second affiliated hospital of guang zhou university of chinese medicine

\section{Hai-rong Cai}

Guangzhou University of Chinese Medicine

\section{Shu-ling Liu}

Guangzhou University of Chinese Medicine

\section{Shuai Zhao}

Guangzhou University of Chinese Medicine

\section{Zhi-shang Li}

Guangzhou University of Chinese Medicine

\section{Si-cong Luo}

Guangzhou University of Chinese Medicine

\section{Xin-gui Cai}

Guangzhou University of Chinese Medicine

\section{Wei-zhang Zhang}

Guangzhou University of Chinese Medicine

\section{Lie-yuan Zhang}

Guangzhou University of Chinese Medicine

Bo-jun Chen ( $\square$ 3021376873@qq.com )

\section{Study protocol}

Keywords: Yiqi Fumai Injection, septic shock, Randomized controlled trial, efficacy, safety

Posted Date: January 24th, 2020

DOI: https://doi.org/10.21203/rs.2.21820/v1

License: @ (i) This work is licensed under a Creative Commons Attribution 4.0 International License. Read Full License 


\section{Abstract}

Background Septic shock is an important problem in critical care medicine and one of the leading causes of death in intensive care units (ICU). In China, Traditional Chinese medicine ( TCM) has been widely used as the adjuvant treatment to improve the symptoms and prognosis in patients with septic shock. Yiqi Fumai Injection ( $\mathrm{YFI}$ ) is one of the most important proprietary TCM for septic shock, previous studies have demonstrated its efficacy and safety. However, these conclusions were limited due to the small sample size and low quality of methodologies of these studies. Therefore, we designed this study to evaluate the efficacy and safety of using YFI as an adjunct treatment for septic shock.

Methods This is a double-blind, randomized, parallel, placebo-controlled clinical trial. A total of 800 participants will be randomly assigned to receive either treatment or placebo in a 1:1 ratio. The treatment group will receive YFI combined with conventional treatment, and the control group will receive $0.9 \%$ sodium chloride injection combined with conventional treatment for 2 weeks. The primary outcome is the 28- days mortality. Secondary outcomes are blood lactate levels, hemodynamics, blood gas analysis, immune function indicators, inflammatory indicators, acute physiology improvement and chronic health assessment (APACHE) II scores, and sepsis-related organ failure score (SOFA). Adverse events will be observed and recorded at the same time for safety assessment.

Discussion This randomized controlled trial will help evaluate the efficacy and safety of YFI for the treatment of septic shock. The results of this trial will provide recommendations for the management of septic shock.

\section{Background}

Sepsis is defined as life-threatening organ dysfunction caused by a dysregulated host response to infection [1]. Septic shock is defined as a subset of sepsis in which underlying circulatory and cellular metabolism abnormalities are profound enough to substantially increase mortality [1]. Currently, it has been estimated to account for more than 19 million new cases

worldwide each year, and 6 million deaths [2].The mortality rate is more than 1/4, and about 3 million of the surviving patients have cognitive dysfunction $[3,4]$.Clinically, approximately $50 \%$ of patients with sepsis progress to septic shock, and the mortality rate of septic shock could be as high as $50 \%$ or more [5-8].Zhou et al [9] showed that the overall hospital mortality rate of patients with severe sepsis/septic shock in the comprehensive intensive care unit in mainland China was 33.5\%. A study in Taiwan, China, found that 28-day mortality rate in patients with severe sepsis/septic shock in the local surgical intensive care unit was $61 \%$ [10].Complex pathogenesis, severe clinical manifestations, and limited treatment made it as one of the leading causes of death in ICU [11-12].Even if survived after treatment, the long-term quality of life is significantly lower than that of the average person [13-15]. Mortality in septic shock has declined over the decades with the rapid advancement of treatments [16-18] .However, with the acceleration of population aging, and the increase of tumor incidence, immunosuppressive drugs, 
antibacterial drugs, invasive treatments, antibiotic resistance rates, the incidence of septic shock is increasing year by year and the number of deaths continues to increase [19-22].Studies have shown that the incidence of septic shock increases at a rate of 1.5-8.0\% per year, the incidence of septic shock increases by $91.3 \%$ in a decade [23-26], and the mortality rate increases with the severity of the disease. Septic shock has led to an increasing economic and public health burden [27-28].

The Surviving Sepsis Campaign 2016 guidelines recommend early resuscitation, antimicrobial therapy, use of vasoactive drugs, glucocorticoid use, anticoagulant therapy, renal replacement therapy, mechanical ventilation, sedation and analgesia, Blood sugar management as conventional treatment for septic shock [29]. Despite such recommendations, the morbidity and mortality rates of septic shock remain high. In addition, adverse reactions and drug tolerance are also ubiquitous. Therefore, there is an urgent need for further efficient and secure management strategies.

In China, traditional Chinese medicine (TCM) has been widely used as an adjuvant therapy for septic shock [30-32]. TCM combined with conventional treatments could relieve symptoms, improve blood gas, and lower blood lactate levels [33-34]. YFI is one of the main proprietary Chinese medicines. A few studies showed that YFI may inhibit inflammation, regulate immune function, and improve patients' Symptoms, reduce the mortality rate [35]. However, these conclusions were limited due to the small sample size and low quality of methodologies of these studies. Therefore, we designed this study to evaluate the efficacy and safety of using YFI as an adjunct treatment for septic shock.

\section{Methods/design}

\section{Setting and design}

This is a randomized, double-blind, parallel, placebo-controlled clinical trial. The protocol was designed using the Consolidated Standards of Reporting Trials (CONSORT) 2010 guidelines and Standard Protocol Items: Recommendations for Interventional Trials (SPIRIT). The study will be conducted at the Second Affiliated Hospital of Guangzhou University of Chinese Medicine. Patients who meet the inclusion criteria criteria for septic shock will be assigned at 1:1 ratio to the treatment group or placebo group. Patients in the treatment group will receive conventional treatment in combination with $\mathrm{YFI}$, and patients in the placebo group will receive conventional treatment combined with $0.9 \%$ sodium chloride injection for 2 weeks, and the post-treatment follow-up period will be 4 weeks. The primary outcome is 28 -days mortality. Secondary outcome includes blood lactate levels, hemodynamics, blood gas analysis, immune function indicators, inflammatory indicators, acute physiology improvement and chronic health assessment (APACHE) II scores, and sepsis-related organ failure score (SOFA) at at baseline, hour 24 , hour 72 and week 2 . Adverse events will be also observed and recorded at the same time for safety assessment. The 14-day mortality rate will be assessed at 14 days after treatment. All patients will be informed about the purpose of the trial, the risks, and the benefits, and informed consent will be obtained from all participants prior to entry into the trial. The data statistics and managers will be blinded. We also 
provide a Clinical Trial Program Specification Guide (SPIRIT) diagram (Figure 1) and a corresponding list (Additional Document 1).

\section{Objectives}

The main objectives of the trial is to assess the efficacy and safety of $\mathrm{YFI}$ as an adjunct treatment in patients with septic shock.

\section{Participants}

\section{Inclusion criteria}

Patients who meet all of the following inclusion criteria will be selected as research volunteers:

(i)All patients met the diagnostic criteria for sepsis. Sepsis is defined as life-threatening organ dysfunction caused by a dysregulated host response to infection. Organ dysfunction can be identified as an acute change in total SOFA score $\geq 2$ points consequent to the infection.

(ii) All patients met the diagnostic criteria for septic shock. Patients with septic shock can be identified with a clinical construct of sepsis with persisting hypotension requiring vasopressors to maintain $\mathrm{MAP} \geq 65 \mathrm{mmHg}$ and having a serum lactate level $>2 \mathrm{mmol} / \mathrm{L}(18 \mathrm{mg} / \mathrm{dL})$ despite adequate volume resuscitation.

(iii) voluntarily agreed to participate by giving written informed consent for the trial;

(iv) man or woman aged between 18 and 70 years old.

\section{Exclusion criteria}

Patients who meet the following criteria will be excluded.

(i)have uncontrollable underlying diseases, such as malignant tumors, active bleeding, definite visceral severe injury, chronic renal failure, chronic liver failure;

(ii)have a history of stress ulcers or severe head injury within 6 months;

(iii) use immunosuppressants or hormones in the prior 3 months;

(iv)age $<18$ years old or $>70$ years old;

(v) allergic to red ginseng, Ophiopogon japonicus, Schisandra and related injection;

(vi) Currently pregnant or breast-feeding;

(vii) Currently participating in another clinical trial; 
(viii)have immunodeficiency diseases.

\section{Ethical aspects}

Ethics approval has been obtained from the Ethics Committee of the Second Affiliated Hospital of Guangzhou University of Chinese Medicine (BF2019-007-01) and registered with the China Clinical Trial Registration Center (ChiCTR-1900026424). The Institutional Review Board will be responsible for overseeing all research procedures, including patient recruitment, random assignment, treatment management, data storage, and more. The study will be conducted in compliance with local law, Declaration of Helsinki, institutional policies and

the Good Clinical Practice (ICH-GCP) guidelines to protect subjects' right and safety. The subject or legal representative will receive sufficient explanation and time consideration before signing the informed consent form. If the patient is unconscious, the right to sign informed consent will be exercised by close relatives who have full capacity for civil conduct. After the patient recovery consciousness, he or she can confirm or withdraw the informed consent form.

\section{Recruitment, screening, and consent}

Recruitment, screening and signing the informed consent will be carried out at the Second Affiliated Hospital of Guangzhou University of Chinese Medicine, and recruitment posters will be distributed in each branch. Once the patient voluntarily participates, the assessment will be based on inclusion criteria and exclusion criteria. Patients who meet all the inclusion criteria and not any exclusion criteria will be provided with and signed the informed consent form prior to the intervention. The recruitment period is 48 months, from June 2019 to June 2023.

\section{Withdrawal or dropout criteria}

Patients will not be included in the analysis under the following ircumstances:

(i)The researchers find that subjects were misdiagnosed as septic shock after randomization.

(ii) The subject did not follow the study plan for medication:

(a) The subject receive the study drug treatment for more than 24 hours;

(b) The subject do not receive the study drug;

(c) subjects receive study drug treatment less than 14 days (excluding discharge from hospital or discharge due to exacerbations or death);

(d) The actual dose is less than $80 \%$ of the total dose (standard rate $<80 \%$ ).

(iii)The lack of necessary data affects the assessment of the primary outcome. 
(iv)The use of banned drugs affects the evaluation of efficacy.

(v) Experiencing anaphylaxis or serious adverse events during the trial.

(vi) Quitting the clinical trial voluntarily.

\section{Discontinuation}

Participants who meet any of the following exclusion criteria will be suspended:

(i) withdrawal of informed consent.

(ii)Serious adverse events (AE) or unacceptable adverse reactions associated with the study drug.

(iii)Serious complications.

(iv) progression of the disease based on the efficacy evaluation criteria and requires urgent treatment.

(v)The regulatory site requires termination of the study.

The reason for the suspension will be recorded on the Case Report Form (CRF), and the last data will be included in the data analysis.

\section{Informed consent}

The researcher reserves the right to fully interpret the content of the manuscript. All subjects will be told to be randomly assigned to the treatment and control groups to clearly communicate possible benefits and risks. Patients can freely withdraw from the study at any time without any penalty or interest loss. However, we will record the reasons for withdrawal and encourage the participants to continue. In the event of withdrawal, the subject's data will not be deleted and will be used for the final analysis.

\section{Sample size}

The sample size was calculated based on the primary endpoint of 28-days mortality. Based on similar previous reports, the 28 -days mortality was $36 \%$ and $25 \%$ for conventional therapy and conventional therapy combined with $\mathrm{YFI}$, respectively. We expect an effect size of at least 0.1 for primary outcomes. A sample size of 332 participants is required to sufficiently detect a target effect size with a type 1 error of $5 \%(\alpha=0.05)$ and $80 \%$ power $(\beta=0.20)$ by using Gpower 3.1.9.2 software. Considering a dropout rate of, a total of 800 participants are necessary, with 400 participants in each group.

\section{Randomization and Blinding}

A statistician from the Second Affiliated Hospital, Guangzhou University of Chinese Medicine GCP Center will generate the randomization code using the SAS 9.2 software analysis system (SAS Institute, Cary, NC, USA). Patients will be randomly divided into the treatment group (YFI combined with conventional 
treatment) and control group ( $0.9 \%$ sodium chloride injection combined with conventional treatment) at the ratio of $1: 1$, whereby the treatment and placebo groups will be coded $A$ and $B$, respectively. Randomization sequences will be concealed in lightproof, sealed envelopes kept by a specified project manager and the sponsor, who are not involved in the recruitment, intervention,

assessment, or statistical analysis. Subjects, nursing staff, laboratory staff and researchers will be blind to grouping and treatment, while drug administrators and dispensing nurses will be aware of grouping information. However, drug managers and dispensing nurses will not be involved in data collection, manage and analysis. The statistician will also be blinded until the data analysis is completed. The YFI and placebo are all dosed with a disposable photophobic infusion set to avoid subject observation of group information. The researchers will accept strict Training, and requires strict adherence to the principle of separation of tasks. The blind codes will not be disclosed until the statistical analysis is completed.

\section{Emergency unblinding}

In cases of medical emergency, the group information will be known and can be obtained from the drug manager. The researcher should contact the person in charge within 24 hours and record the information in $\mathrm{CRF}$, including reasons for unblinding date, medical emergency, treatment and results.

\section{Intervention}

After randomization, eligible participants will be randomized to either the

treatment or control group, receiving intravenous injection of either $\mathrm{YFI}(0.6 \mathrm{gmg} / \mathrm{bottle}, 8 \mathrm{bottles} / \mathrm{box})$ or placebo $(0.9 \%$ sodium chloride injection, $250 \mathrm{~mL} / \mathrm{bag})$ once daily for 2 weeks. YFI was manufactured by Tianjin Tianshili Zhijiao Pharmaceutical Company(Tianjin, China) (batch number: 20190033). 0.9\% sodium chloride injection is provided by the pharmacy of the Second Affiliated Hospital of Guangzhou University of Traditional Chinese Medicine. Both groups will receive conventional treatment recommended by the the Surviving Sepsis Campaign 2016 guidelines, including early resuscitation, antimicrobial therapy, use of vasoactive drugs, glucocorticoid use, anticoagulant therapy, renal replacement therapy, mechanical ventilation, sedation and analgesia, and blood sugar management.

The treatment group will receive routine medication combined with intravenous injection of YFI $5.2 \mathrm{~g}+$ $0.9 \%$ sodium chloride injection $250 \mathrm{~mL}$ for 120 minutes, once daily. The control group will receive routine medication combined with intravenous injection of $0.9 \%$ chlorine Sodium injection $250 \mathrm{~mL}$ intravenous for 120 minutes. The dose and speed of the two groups were the same. Patients will begin drug intervention within 24 hours of recruitment with a period of 2 weeks. The disposable photophobic infusion set will be used, so that YFI and placebo have the same appearance and color, all drugs are uniformly packaged and use the same identification label.

Tianjin Tianshili Zhijiao Pharmaceutical Company stated that there is no conflict related to the benefits of this research. The quality of the drug is in compliance with the Chinese Medicine Standards State Food 
and Drug Administration (SFDA). Any other Chinese herbal medicine soup or proprietary Chinese medicine will be banned in this trial.

\section{Precautions}

(i) Chinese medicine injection, Chinese medicine, acupuncture and other Chinese medicine treatments are prohibited during the study period.

(ii)Routine treatment of septic shock should be performed, and YFI should not be used as an alternative treatment.

(iii) If the patient is discharged from the hospital within 2 weeks because of improvement, or other treatment is needed when condition is aggravated, the use of YFI will be suspended.

\section{Outcome measures}

\section{Primary outcome measures}

The primary outcome is 28-days mortality.

\section{Secondary outcome measures}

The secondary outcome indicator will be:

(i)Changes in immune index: T lymphocyte subset typing, neutrophil cd64 percentage.

(ii)Changes in inflammatory markers: C-reactive protein(CRP), procalcitonin(PCT), interleukin-6(IL-6),

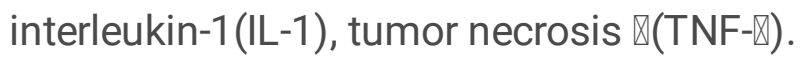

(iii)Blood gas analysis: oxygenation index (PaO2/FiO2), blood gas, blood lactate.

(iv) Hemodynamics: heart rate (HR), mean arterial pressure (MAP), central venous pressure (CVP) and cardiac index $(\mathrm{Cl})$.

(v)Sepsis-related organ failure score (SOFA).

(vi)Improve acute physiology and chronic health assessment II (APACHE-II) scores;

(vii)The name, dose and duration of use of the vasoactive drug.

(viii)The name, dose and duration of corticosteroid use.

(x)The incidence of complications.

(xi) The duration and cost of ICU.

(xii)Hospitalization time and expenses. 


\section{Follow up}

Various parameters were collected based on time points (Table 1).

(i) Baseline (1 day): 24 hours before recruitment.

(ii)Treatment period (within 14 days after intervention): 24h, 72h,7days and 14 days after intervention.

(iii)Follow-up period (within 28 days after intervention): 28-days mortality was performed on 28.

If the patient is discharged from the hospital, contact by phone or text message.

\section{Safety and adverse events monitoring}

Safety and Adverse Event will be monitored by an independent data security and surveillance committee (DSMB), consisted of clinicians, evidence-based medical experts, and statisticians. Major vital signs, physical examinations, and some laboratory tests will be performed daily for safety assessment. The main vital signs include body temperature, blood pressure, heart rate and respiratory rate. Laboratory tests include routine blood, urine and stool tests, and fecal occult blood tests; electrocardiographic liver function tests (alanine aminotransferase (ALT), aspartate aminotransferase (AST), alkaline phosphatase (ALP), gamma- Glutamyltranspeptidase (GGT) and serum total bilirubin (TBIL); renal function tests (serum creatinine $(\mathrm{Cr})$ and blood urea nitrogen $(\mathrm{BUN}))$; and electrolyte tests $(\mathrm{K}+, \mathrm{Na}+, \mathrm{Cl}$ - and $\mathrm{Ca} 2+)$.

DSMB specifies the severity of adverse events as mild, moderate, severe or serious adverse events. Any adverse events during the the study should be recorded, including time, severity, duration of adverse events, treatment measures and outcomes. Subjects with adverse events will be followed up until the adverse events disappear and the laboratory indicators return to normal or baseline levels. The relationship between adverse events and drugs is assessed as "impossible", "suspicious", "possible", "possible". AE will be reported to the Principal Investigator and the Ethics Reporting Committee to determine Whether it should be withdrawn from the trial. If any symptoms worsen, the patient will be withdrawn from the study and referred to further treatment.

Quality control

In order to ensure the quality of the study, the data will be imported into the ResMan website in time for release. The Clinical Drug Research Center of Guangzhou University of Chinese Medicine will serve as a data monitoring team to identify problems in the project, control bias, and ultimately decide to terminate the research. Clinical trial specialists will be invited to monitor the trial. Researchers will be trained to ensure the quality of the trial. Clinicians and nurses participating in this trial have 5-10 years of experience. We will conduct three intensive trainings for these researchers before the start of the trial.

supervision and inspection 
This study will be subject to regulatory supervision and inspection every 2 weeks, including data integrity, informed consent, inclusion and exclusion criteria, raw data, handling of adverse events, storage conditions and destruction.

\section{Compliance control}

Researchers should undergo rigorous training, and trainers must have sufficient clinical research experience and pass the " Good Clinical Practice Pharmaceutical Products (GCP)" by the National Food and Drug Administration (FDA) certification to ensure the quality of the training. Researchers should read and understand the details of the trial and explain the potential benefits or adverse reactions to obtain the subject's informed consent.

\section{Statistical analysis}

All Outcomes will be conducted based on the intention-to-treat (ITT) principle. Missing values will be imputed by the last-observation carried-forward method. The statistical analysis will be performed using the SPSS 21.0(IBM Corp., Armonk, NY, USA). Baseline characteristics will be summarized by means of simple descriptive statistics. The two sample Student's $t$ test will be used for continuous variables and the chi-square test or Wilcoxon test will be

used for categorical variables. The primary analysis will be a comparison of 28-days mortality, the chisquared test or Fisher's exact test will be used.

For secondary outcome measures, repeated measures analysis of variance (ANOVA) will be applied to determine changes at each visit to investigate the effects of treatment and time course. Within-group differences will be assessed with a paired $t$ test for normally distributed data and a Wilcoxon signed-rank test for non-normally distributed data. For AE evaluation, all AEs will be reported including its grade, time of occurrence, duration, treatment, prognosis, and correlation with the intervention drugs. The chi-square test or Fisher exact test will be used to compare the incidence of AEs between the two groups.

\section{Data management}

In this trial, we will use two data entry systems. The researcher will fill the information in CRF and enter it into the electronic version of CRF in time, then submit it to research assistant after checking. Time, the person and the reason for the revision will be record when correction is needed. The research assistant is responsible for verifying the consistency and accuracy of the paper and electronic of CRF and providing feedback to the clinical researchers. After the trial is completed and the blind is disclosed, the data will be locked and can not be modified afterwards. The data manager should document and maintain the CRF. The medical information of subject is confidential and must not be disclosed to a third parties.

\section{Discussion}


There is still a high incidence and mortality in septic shock, which lead to serious economic and social burdens. TCM was first recorded about 2,500 years ago and more and more evidences prove the effect of Chinese and Western medicine for septic shock. Hence, Chinese medicine can be used as an adjuvant treatment of septic shock to obtain better results. YYFI has been widely used to treat septic shock in China. The YFI consists of three herbs: red ginseng, Liriope graminifolia and Schisandra chinensis. Previous studies have shown that YFI could improve blood gas, lower lactate levels and improve symptoms. However, these conclusions were limited due to the small sample size and low quality of methodologies of these studies. Therefore, we designed this study to evaluate the efficacy and safety of using YFI as an adjunct treatment for septic shock. This study was designed as a randomized, doubleblind, parallel, placebo-controlled trial that will provide strong evidence of the efficacy and safety of YFI for the treatment of septic shock. However, this study has certain limitations. First, because the study is being conducted in Guangdong, China, the relative role of uncertain test drugs will be similar in other races. Second, the follow-up time is relatively short.

\section{Abbreviations}

ICU: intensive care units, TCM: Traditional Chinese medicine, YFI: Yiqi Fumai Injection, APACHE-II: acute physiology improvement and chronic health assessment II scores, SOFA: sepsis-related organ failure score, AE: adverse events, CONSORT: Consolidated Standards of Reporting Trials, SPIRIT: Standard Protocol Items: Recommendations for Interventional Trials, MAP: mean arterial pressure, ICH-GCP: institutional policies and the Good Clinical Practice, ALT: alanine aminotransferase, AST: aspartate aminotransferase, ALP: alkaline phosphatase, GGT: gamma- Glutamyltranspeptidase, TBIL: serum total bilirubin ,Cr: serum creatinine, BUN: blood urea nitrogen, HR: hemodynamics heart rate, CVP: central venous pressure, Cl: cardiac index, CRP: C-reactive protein, PCT; procalcitonin, IL-6冈interleukin-6, IL-1凹

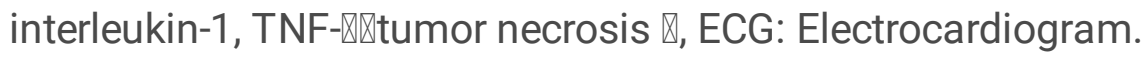

\section{Declarations}

\section{Trial status}

The trial protocol is version 1.0, dated 14/3/2019. Recruitment started in November 2019 and is expected to be completed in November 2023.

\section{Additional files}

Additional files 1区The SPIRIT checklist.

\section{Dissemination policy}

Results will be published in peer-reviewed journals and presented at national and international scientific meetings regardless of the magnitude or direction 
of effect.

\section{Ethics approval and consent to participate}

The trial is in line with the Helsinki Declaration and China Clinical Trial Quality Management Regulations. This trial has been approved by the Ethics Committee of the Second Affiliated Hospital of Guangzhou University of Chinese Medicine (BF2019-007-01) and has been registered in China Clinical Registration Center (ChiCTR-1900026424). All subjects will receive written informed consent prior to enrollment.

\section{Consent for publication}

Not applicable. All the participants whose identifiable details are included in the manuscript approved the final manuscript and consent for publication

\section{Availability of data and materials}

Not applicable. All data are fully available without restriction.

\section{Competing interests.}

The authors declare that they have no competing interests.

\section{Funding}

The study was funded by Tianjin Tianshili Zhijiao Pharmaceutical Company. The funder provided research funding but had no role in the in the design of the study and collection, analysis, and interpretation of data and in writing the manuscript.

\section{Authors'contributions}

Jing Zeng conceived and designed a forward-looking experiment and drafted a manuscript. Hai-rong Cai and Shu-ling Liu helped design the forward-looking experiment, drafted the manuscript. Shuai Zhao helped draft the manuscript. Zhi-shang Li helped to revise the prospective trial. Si-cong Luo determined the statistical analysis plan. Xin-gui Cai calculates the sample size and participates in statistical analysis. Wei-zhang Zhang helps data collection and data management. Lie-yuan Zhang is responsible for the quality control of the test. Bo-jun Chen monitored the progress and quality control. All authors have read and approved the final manuscript.

\section{Acknowledgements}

Thanks to Yan-hong Chen of Guangzhou University of Chinese Medicine for her help.

\section{Authors' information (optional)}

${ }^{1}$ The Second Affiliated Hospital, Guangzhou University of Chinese Medicine, Guangzhou 510006, China. 
${ }^{2}$ The second clinical medical school, Guangzhou University of Chinese Medicine, Guangzhou 510405, China.

\section{References}

[1]Singer M , Deutschman C S , Seymour C W , et al. The Third International Consensus Definitions for Sepsis and Septic Shock (Sepsis-3)[J]. The Journal of the American Medical Association, 2016, 315(8):775-787.

[2] Perner A , Cecconi M , Cronhjort $M$, et al. Expert statement for the management of hypovolemia in sepsis[J]. Intensive Care Medicine, 2018,44(6):791-798.

[3] Prescott H C, Angus D C. Postsepsis Morbidity.[J]. Jama, 2018, 319(1):91.

[4] Reinhart K, Daniels R, Kissoon N, et alهRecognizing Sepsis as a Global Health Priority-A WHO Resolution [J]. New England Journal of Medicine,2017, 377(5):414-417.

[5] Kaukonen K M, Bailey M, Suzuki S, et al. Mortality related to severe sepsis and septic shock among critically ill patients in Australia and New Zealand, 2000-2012.[J]. Journal of the American Medical Association, 2014, 311(13):1308.

[6] Landesberg G, Dan G, Meroz Y, et al. Diastolic dysfunction and mortality in severe sepsis and septic shock[J]. European Heart Journal, 2012, 33(7):895.

[7] Huang C T , Chuang Y C , Tsai Y J , et al. High Mortality in Severe Sepsis and Septic Shock Patients with Do-Not-Resuscitate Orders in East Asia[J]. PLOS ONE, 2016, 11(7):e0159501.

[8] Balk R A. Severe sepsis and septic shock. Definitions, epidemiology, and clinical manifestations.[J]. Critical Care Clinics, 2000, 16(2):179-192.

[9] Zhou J , Qian C , Zhao M , et al. Epidemiology and Outcome of Severe Sepsis and Septic Shock in Intensive Care Units in Mainland China[J]. PLoS ONE, 2014, 9(9):e107181.

[10] Huang C T , Tsai Y J , Tsai P R, et al. Epidemiology and Outcome of Severe Sepsis and Septic Shock in Surgical Intensive Care Units in Northern Taiwan[J]. Medicine, 2015, 94(47):e2136.

[11] Boussekey N, Cantrel J, Debrabant L D, et al. Epidemiology, Prognosis, and Evolution of Management of Septic Shock in a French Intensive Care Unit: A Five Years Survey[J]. Critical Care Research \& Practice, 2010, 2010(6):436427.

[12] Alberti C, Brunbuisson C, Burchardi $\mathrm{H}$, et al. Epidemiology of sepsis and infection in ICU patients from an international multicentre cohort study[J]. Intensive Care Medicine, 2002, 28(4):525-526. 
[13] Schoenberg M H, Weiss M, Radermacher P. Outcome of patients with sepsis and septic shock after ICU treatment[J]. Langenbecks Archives of Surgery, 1998, 383(1):44-48.

[14] Riché F, Chousterman B G, Valleur P, et al. Protracted immune disorders at one year after ICU discharge in patients with septic shock:[J]. Critical Care, 2018, 22(1):42.

[15] Granja C, Dias C, Costapereira A, et al. Quality of life of survivors from severe sepsis and septic shock may be similar to that of others who survive critical illness[J]. Critical Care, 2004, 8(2):R91-R98.

[16] Stoller J, Halpin L, Weis M, et al. Epidemiology of severe sepsis: 2008-2012.[J]. Journal of the American College of Surgeons, 2016, 31(1):58-62.

[17] Martin G S , Mannino D M , Eaton S , et al. The Epidemiology of Sepsis in the United States from 1979 through 2000[J]. New England Journal of Medicine, 2003, 348(16):1546-1554.

[18] Harrison D A, Welch C A, Eddleston J M. The epidemiology of severe sepsis in England, Wales and Northern Ireland, 1996 to 2004: secondary analysis of a high quality clinical database, the ICNARC Case Mix Programme Database[J]. Critical Care, 2006, 10(2):R42-R42.

[19] Cheng B , Xie G , Yao S L , et al. Epidemiology of severe sepsis in critically ill surgical patients in ten university hospitals in China*[J]. Critical Care Medicine, 2007, 35(11):2538-2546.

[20] Annane D , Aegerter $P$, Jars-Guincestre M C , et al. Current epidemiology of septic shock: the CUB-Réa Network[J]. Am J Respir Crit Care Med, 2003, 168(2):165-172.

[21] Quenot J P, Binquet C, Kara F, et al. The epidemiology of septic shock in French intensive care units: the prospective multicenter cohort EPISS study.[J]. Critical Care, 2013, 17(2):R65.

[22] Gray A, Ward K, Lees F, et al. The epidemiology of adults with severe sepsis and septic shock in Scottish emergency departments[J]. Emergency Medicine Journal, 2013, 30(5):397-401.

[23] Finfer S , Bellomo R , Lipman J , et al. Adult-population incidence of severe sepsis in Australian and New Zealand intensive care units[J]. Intensive Care Medicine, 2004, 30(4):589-596.

[24] Jesús Blanco, Arturo Muriel-Bombín, Víctor Sagredo, et al. Incidence, organ dysfunction and mortality in severe sepsis: a Spanish multicentre study[J]. Critical Care, 2008, 12(6)囚R158.

[25] Rangel-Frausto M S , Pittet D , Costigan M , et al. The Natural History of the Systemic Inflammatory Response Syndrome (SIRS)[J]. JAMA, 1995, 273(2):117.

[26] Adrie C , Alberti C , Chaix-Couturier C , et al. Epidemiology and economic evaluation of severe sepsis in France: age, severity, infection site, and place of acquisition (community, hospital, or intensive care unit) as determinants of workload and cost[J]. Journal of Critical Care, 2005, 20(1):46-58. 
[27] Soares M O, Welton N J, Harrison D A, et al. Intravenous immunoglobulin for severe sepsis and septic shock: clinical effectiveness, cost-effectiveness and value of a further randomised controlled trial[J]. Critical Care, 2014, 18(6):1-13.

[28] Huang D, Angus D, Dremsizov T, et al. Cost-effectiveness of early goal-directed therapy in the treatment of severe sepsis and septic shock[J]. Critical Care, 2003, 7(S2):1-1.

[29] Rhodes A, Evans L E, Alhazzani W, et al. Surviving Sepsis Campaign: International Guidelines for Management of Sepsis and Septic Shock: 2016[J]. Critical Care Medicine, 2017, 43(3):486-552.

[30] Ma H, Kou J, Zhu D, et al. Liu-Shen-Wan, a traditional Chinese medicine, improves survival in sepsis induced by cecal ligation and puncture via reducing TNF-a levels, MDA content and enhancing macrophage phagocytosis[J]. International Immunopharmacology, 2006, 6(8):1355-1362.

[31] Lin S J, Cheng Y Y, Chang C H, et al. Traditional Chinese Medicine Diagnosis "Yang-Xu Zheng": Significant Prognostic Predictor for Patients with Severe Sepsis and Septic Shock[J]. Evidence-Based Complementray and Alternative Medicine,2013,(2013-10-24), 2013, 2013(2013):759748.

[32] Zhi Y, Wang K, Wang L. [Interpretation of the section of Chinese herb medicine in "Chinese guidelines for management of severe sepsis and septic shock 2014"].[J]. Zhonghua Wei Zhong Bing Ji Jiu Yi Xue, $2015,27(6): 429$.

[33] Chen Z G, Luo H, Xu S, et al. Study on the methodology of developing evidence-based clinical practice guidelines of chinese medicine[J]. Chinese Journal of Integrative Medicine, 2015, 21(11):874-880.

[34] Liang X, Zhou M, Ge X Y, et al. Efficacy of traditional Chinese medicine on sepsis: a systematic review and Meta-Analysis[J]. International Journal of Clinical \& Experimental Medicine, 2015, 8(11):20024.

[35] Xie R F. [Effects of Early Goal-directed Therapy Combined with Yiqi Fumai Lyophilized Powder on $\mathrm{P}(\mathrm{cv}-\mathrm{a}) \mathrm{CO} 2$ and S(cv)O2 in Patients with Septic Shock][J]. Zhongguo Zhong xi yi jie he za zhi Zhongguo Zhongxiyi jiehe zazhi $=$ Chinese journal of integrated traditional and Western medicine $/$ Zhongguo Zhong xi yi jie he xue hui, Zhongguo Zhong yi yan jiu yuan zhu ban, 2016, 36(3):306.

\section{Tables}

Table 1 Measurement items and points of data capture 


\begin{tabular}{|c|c|c|c|c|c|c|}
\hline & \multirow[t]{2}{*}{ Baseline } & \multicolumn{4}{|c|}{ Treatment period } & \multirow{2}{*}{$\begin{array}{l}\text { Follow-up } \\
\text { Period }\end{array}$} \\
\hline & & $24 \mathrm{~h}$ & $72 \mathrm{~h}$ & 7 days & 14 days & \\
\hline \multicolumn{7}{|l|}{ Enrollment } \\
\hline Informed consent & $x$ & & & & & \\
\hline Inclusion/exclusion criteria & $x$ & & & & & \\
\hline Medical history & $x$ & & & & & \\
\hline Allocation & $x$ & & & & & \\
\hline \multicolumn{7}{|l|}{ Intervention } \\
\hline \multicolumn{7}{|l|}{ Assessments } \\
\hline Concomitant medication & $x$ & $x$ & $x$ & $x$ & $x$ & \\
\hline vital signs & $x$ & $x$ & $x$ & $x$ & $x$ & \\
\hline 12-lead ECG & $x$ & & & & $x$ & \\
\hline liver function and renal function tests & $x$ & & & & $x$ & \\
\hline Electrolyte tests & $x$ & & & & $x$ & \\
\hline T lymphocyte subset typing and neutrophil CD64 percentage. & $\times$ & $x$ & $x$ & $\times$ & $x$ & \\
\hline CRP,PCT,IL-6, IL-1,TNF- & $x$ & $\times$ & $\times$ & $\times$ & $x$ & \\
\hline $\mathrm{PaO} 2 / \mathrm{FiO} 2$, blood gas, blood lactate & $\times$ & $\times$ & $\times$ & $\times$ & $\times$ & \\
\hline HR, MAP,CVP and CI & $x$ & $x$ & $x$ & $x$ & $x$ & \\
\hline SOFA & $x$ & $x$ & $x$ & $x$ & $x$ & \\
\hline APACHE-II & $x$ & $x$ & $x$ & $x$ & $x$ & \\
\hline The name, dose and duration of use of the vasoactive drug & & $x$ & $x$ & $x$ & $x$ & \\
\hline The name, dose and duration of corticosteroid use & & $x$ & $x$ & $x$ & $x$ & \\
\hline Adverse event evaluation & & $x$ & $x$ & $x$ & $x$ & \\
\hline The duration and cost of ICU & & & & & $x$ & \\
\hline 28-days morality & & & & & & $x$ \\
\hline
\end{tabular}

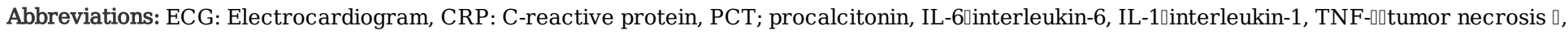
HR: hemodynamics heart rate, CVP: central venous pressure, CI: cardiac index, MAP: mean arterial pressure, APACHE-II: acute physiology improvement and chronic health assessment II scores, SOFA: sepsis-related organ failure score,

PaO2/FiO2:Arterial oxygen partial pressure/ Inhaled oxygen concentration.

\section{Figures}




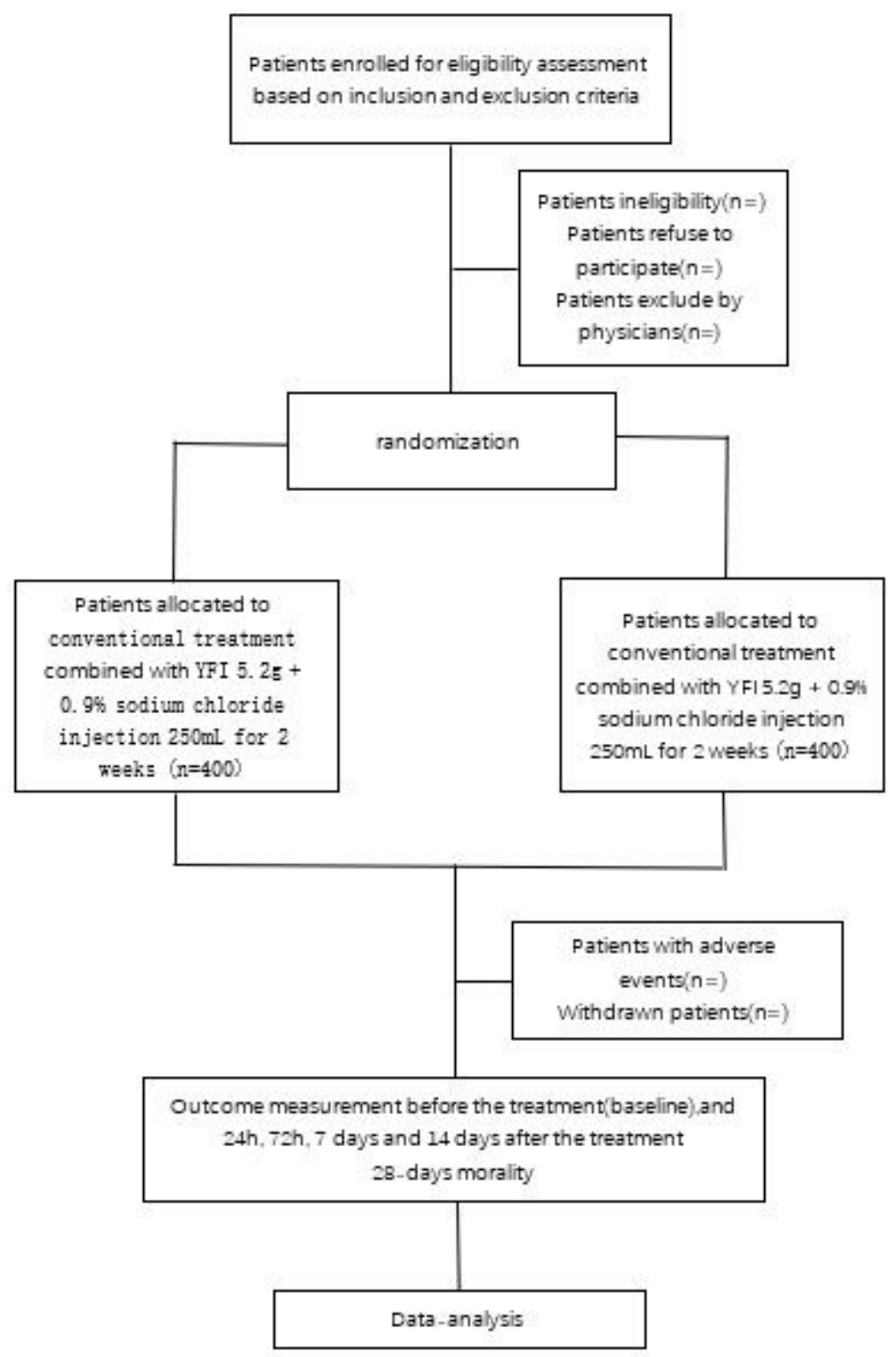

Figure 1

Study flow diagram

\section{Supplementary Files}

This is a list of supplementary files associated with this preprint. Click to download.

- TheSPIRITchecklist.docx 